\title{
Competencias cognitivas en el diseño de materiales educativos multimedia del docente de contextos educativos virtuales
}

\author{
Cognitive competences in the design of multimedia educational materials of \\ the teacher of virtual educational contexts
}

$\begin{array}{lr}\text { Florángel Chacón B. } & \text { Artículo recibido en febrero } 2019 \\ \text { florangelch@gmail.com } & \text { Arbitrado en marzo } 2019 \\ \text { Código ORCID: 0000-0003-4330-041x } & \text { Publicado en mayo } 2019 \\ \text { Universidad Pedagógica } & \\ \text { Experimental Libertador, Venezuela } & \end{array}$

\section{Resumen}

El propósito del trabajo fue describir las competencias cognitivas en el manejo y uso de las TIC de los profesores del programa de especialización en telemática e informática en educación a distancia de la Universidad Nacional Abierta (UNA). La investigación fue de tipo descriptiva, con diseño de campo, transeccional contemporáneo y una amplitud de foco unieventual. La técnica utilizada para la recolección de datos fue la encuesta y como instrumento el cuestionario. Para la validez se utilizó la técnica de juicio de expertos, llevado a cabo por profesores adscritos a otros programas de postgrado de la UNA. La unidad de estudio la conformaron ocho profesores adscritos a la Dirección de Investigaciones y Postgrado como subsistema de la UNA. Los resultados que caracterizan la formación docente arrojaron un proceso reflexivo importante orientado hacia una necesaria renovación de las competencias de los docentes.

\begin{abstract}
The purpose of the work was to describe the cognitive competences in the management and use of ICT of the professors of the specialization program in telematics and informatics in distance education of the National Open University (UNA). The research was descriptive, with a field design, contemporary transectional and a one-event width of focus. The technique used for data collection was the survey and the questionnaire as an instrument. For validity, the expert judgment technique was used, carried out by professors assigned to other UNA graduate programs. The study unit was made up of eight professors attached to the Research and Postgraduate Directorate as a subsystem of UNA. The results that characterize teacher training produced an important reflective process oriented towards a necessary renewal of teachers' competences.
\end{abstract}

Palabras clave:

Materiales educativos multimedia; TIC;

formación docente virtual; estrategias

Keywords:

Multimedia educational materials; TIC; virtual teacher training; strategies 
INTRODUCCIÓN

La sociedad de la información y de la comunicación plantea constantes retos. Uno de ellos gira en torno a procesos formativos que permitan la gestión de información de manera efectiva para producir conocimientos que puedan ser significativos y compartidos. Esta gestión e intercambio de información demanda el uso de tecnología de vanguardia, a la luz del vertiginoso avance de las tecnologías de la información y de la comunicación (TIC).

Las TIC se han incorporado en el diario quehacer del hombre y han revolucionado todo ámbito de la existencia y, por supuesto, han impactado los procesos de enseñanza y aprendizaje en todos los niveles educativos. Ahora bien, dicha incorporación no puede darse en ritmos de tiempo muy aletargados, más aún cuando los estudiantes, producto de su curiosidad, presentan mayores niveles de uso de las tecnologías.

Tal situación despierta el interés e invita a la revisión y cambio constante en aquellos aspectos o elementos del proceso educativo que puedan ser objeto de transformación y/o actualización a través del uso de tales tecnologías. Específicamente a nivel universitario en la modalidad a distancia, el tema de la calidad didáctica de los materiales educativos reviste significado especial, por cuanto es a través de ellos que se facilita el aprendizaje.

Los materiales representan un apoyo, una orientación, una invitación al desarrollo de habilidades de orden superior tal como la reflexión y análisis de situaciones. Son recursos que utiliza el estudiante para interactuar, para aprender, para extraer información clave que le permita resolver situaciones, dialogar con sus compañeros, con sus tutores.

En razón de lo planteado, se hace necesario que el docente tengo entre sus competencias el conocer los aspectos básicos para diseñar, desarrollar, e implementar en sus aulas de clases materiales educativos innovadores, desarrollados con tecnología apoyada en la web y que potencien la educación que reciben los estudiantes

La Educación en su fin último se relaciona con desarrollo, crecimiento, evolución, incluso con sostenimiento de patrones de la sociedad. Representa ésta una potente herramienta no solo para el crecimiento del ser humano sino para la sociedad en la cual se encuentra inmerso.

Tal como es señalado por Mayor en su prólogo en la Conferencia Mundial de Educación Superior de la UNESCO (1998), donde expone que la educación "está también llamada a contribuir a la solución de los grandes problemas de alcance mundial, regional y local (pobreza, exclusión, agravación de las desigualdades, deterioro del medioambiente, etc.)" (p. 4).

La educación juega un papel importante en la humanidad y como tal debe adaptarse para cubrir las necesidades presentes en ella. Producto de los exponenciales cambios en el ámbito tecnológico la educación enfrenta un interesante reto: incorporar el uso de las TIC en aras de fortalecer los aprendizajes, lo cual generaría un proceso educativo de manera innovadora, motivadora y creativa; y lo cual se alcanza a través de una pedagogía emergente que involucre propuestas curriculares adaptadas al uso de las tecnologías, con estrategias didácticas y recursos educativos que potencien el acto formativo.

Acto formativo que se desarrolla en un espacio rico en conocimientos, de posibilidades y de interactividad e interacción que promueven el surgimiento de competencias diferenciadoras en sus participantes.

Tal como se establece en el primer artículo de la Declaración mundial sobre la 
educación superior en el siglo XXI: Visión y Acción UNESCO (1998):

formar [...] ciudadanos responsables, capaces de atender a las necesidades de todos los aspectos de la actividad humana, ofreciéndoles cualificaciones que estén a la altura de los tiempos modernos, comprendida la capacitación profesional, en las que se combinen los conocimientos teóricos $y$ prácticos de alto nivel mediante cursos y programas que estén constantemente adaptados a las necesidades presentes y futuras de la sociedad. (p. 2)

La Educación Superior se orienta hacia temas de profesionalización, de especialización, de distribución de saberes para la inserción de la persona al campo laboral con competencias que logren un óptimo desempeño en el área respectiva que a su vez responda a demandas sociales, y permita resolver problemas a través de la investigación, de la innovación y generación de conocimientos que se brindan en los recintos universitarios.

De igual manera, las universidades, a la par que la educación, promueven el desarrollo económico, social y cultural de un país, al conjugarse en sus recintos la dualidad creación y transmisión de conocimientos. Además, estas instituciones permiten el cumplimiento del derecho fundamental de las personas a la educación, y el estado las involucra dentro de sus políticas sociales, en específico, en cuanto al promover el acceso a la educación superior.

Entre los años 6o-7o, al igual que en la segunda mitad del siglo XX, la demanda estudiantil para realizar estudios de cuarto nivel tuvo un crecimiento exponencial, Guadilla (2005), hace referencia a este crecimiento así: "desde el punto de vista cuantitativo... como la época de la expansión más espectacular de los sistemas de educación superior. El número de estudiantes matriculados en todo el mundo se multiplicó por más de seis veces..." (p. 14).

En Venezuela, la tasa promedio de incremento de matrícula en la educación superior para la década de los setenta fue de un $15,75 \%$ anual. La demanda para la continuación de estudios a nivel superior superó la capacidad de ingreso que tenían las universidades para el momento, lo que evidenció, según se expresa en la Conferencia Mundial sobre la Educación Superior de la UNESCO, la desventaja real en la que se encontraba la población de países menos desarrollados, en cuanto a la oferta de estudios de cuarto nivel.

La situación antes descrita hizo que se fijara la mirada hacia la educación a distancia como modalidad de estudios, que como bien su nombre indica presenta como principal característica la distancia. La educación a distancia como modalidad ofrece alternativas y posibilidad real de acceso a un grupo de la población que no continuaron estudios superiores, por diversas razones: escasa oferta, falta de tiempo, jornadas laborales extensas, sumado a factores propios del ámbito familiar y social, ubicación geográfica (poblaciones lejanas a los centros educativos) e incluso razones orientadas hacia temas económicos y capacidades físicas, superando barreras de espacio y de tiempo.

De esta forma en Venezuela, bajo el esquema de universidad experimental, se eleva la educación a distancia a la categoría universitaria, al crear la Universidad Nacional Abierta, como universidad pionera en esta modalidad de estudio en América Latina. Dicha institución de educación superior se concibió para la formación de profesionales en áreas prioritarias del desarrollo social, se organizó como un sistema de educación abierto y a distancia, 
de alcance nacional y proyección internacional, con el enfoque de democratizar y masificar el acceso a una educación, permanente, de calidad y comprometida con el desarrollo del país.

Desde sus inicios dicha Universidad ha utilizado diferentes formatos tecnológicos para abordar el acto educativo, además de permitir ampliar la cobertura para la participación de quien requiera educación, así como cumplir con los objetivos establecidos por la UNESCO (2000) en cuanto educación para todos, utilizando como estrategia las TIC.

Las TIC se configuran como tecnologías que, acompañadas de efectivas estrategias didácticas, potencian un aprendizaje flexible, propician autonomía en el estudiante, lo llevan a asumir la responsabilidad de su proceso, además de permitir romper barreras de espacio y de tiempo, desapareciendo fronteras.

Asimismo, brindan al estudiante un espacio interactivo interesante y de manera permanente, además del acceso a información y uso de bibliotecas digitales y de otros servicios en la red que en épocas anteriores resultaban de difícil hasta impensable acceso.

Otro aspecto que cobra importancia cuando se incorpora las TIC en la modalidad educativa a distancia es que permiten añadir un ingrediente innovador en el proceso de aprendizaje de la persona, al incluir herramientas que responden a diferentes estilos de aprendizaje. De igual manera, con el uso de las TIC se capta la atención de los estudiantes de manera diferente y se logra un mayor nivel de personalización y adecuación a las necesidades formativas del individuo.

Sin embargo, el solo hecho de utilizar las TIC no es suficiente, se requiere abordar el tema de la brecha digital en cuanto acceso a recursos informáticos que le permitan al individuo sumergirse en esta modalidad de estudio sin inconveniente alguno, no solo en cuanto a disponibilidad de dispositivos electrónicos sino a la posibilidad de conexión por servicios que puedan ofrecerles según anchos de bandas propios de la ciudad, estado o país donde se encuentre la persona, lo que puede producir letargos en la transmisión y recepción de información.

En el caso del diseño de los materiales educativos multimedia estos constituyen el medio para el desarrollo del aprendizaje y enlace entre el docente, los contenidos, los estudiantes e incluso la institución. Sin embargo, en la institución objeto de este estudio se evidencia según lo observado en las aulas virtuales del programa un enfoque lineal, lo cual se refleja en el uso de materiales educativos para la transmisión de contenidos, con un enfoque conductista y una evaluación centrada hacia la tutoría automática, lo cual hace que se deje de lado los espacios para el intercambio entre docente y el estudiante, con diseños enfocados hacia la individualidad con actividades reiterativas, lo cual limita la construcción del aprendizaje.

Lo antes planteado causa desmotivación, disminución en los índices de prosecución de la carrera de los estudiantes, adicionalmente deja de lado el uso de medios actualizados que añaden dinamismo, podrían incrementar la motivación y aporta elementos innovadores que situarían como de vanguardia dichos estudios de postgrado, no solo por sus contenidos, tutoría, sino por sus estrategias diferentes materiales educativos multimedia que podrían utilizarse.

Asimismo, al tomar como referente la dimensión objetiva de la calidad didáctica, se evidencia al revisar los planes de estudio y por conversaciones con los profesores que dictan asignaturas en uno de los programas de postgrado de la UNA, como también de mensajes recibidos por los participantes en los programas, y de la experiencia propia del 
autor de esta investigación, se constata que los materiales educativos utilizados en las diferentes estrategias de enseñanza $y$ aprendizaje de las asignaturas fueron desarrollados con tecnologías que los sitúan en un determinado período de la línea del tiempo.

Tales materiales eran perfectamente apropiados en su momento, alcanzando resultados extraordinarios. Ahora bien, como parte integral de las funciones que tiene el docente, se destaca en sus actividades, la planificación de su proceso de enseñanza, momento en el cual el docente asume el liderazgo en cuanto a la organización y presentación de contenidos a los estudiantes, definición de estrategias didácticas, entre otras.

Obviamente, para lograrlo el docente se requiere de herramientas, conocimientos y destrezas; así como, considerar los diferentes recursos metodológicos y didácticos disponibles que pongan de manifiesto su actitud hacia la innovación de manera tal que le permitan lograr, con dotes diferenciadores, sus objetivos de enseñanza para catalizar a su vez los objetivos de aprendizaje.

Lo expuesto supone una formación inicial y continúa del profesor, más enfocada hacia el diseño de experiencias formativas en contextos de aprendizaje con el uso de las TIC, en la mediación y tutorización, y en las estrategias instruccionales con el diseño de sus respectivos materiales didácticos, propias de ambientes virtuales, puesto que su sitio de trabajo son aulas virtuales.

Sin embargo, en el caso de la Universidad Nacional Abierta, la formación del docente no ha sido continúa en cuanto a ofrecimiento de cursos o programas de actualización. Se tiene como evidencia que, en los últimos años, no se presentan iniciativas orientadas hacia la formación y capacitación de su talento humano en tales temas por parte de la institución.
En este contexto, enmarcados en la calidad educativa de los materiales didácticos como eje motor del proceso de enseñanzaaprendizaje en la modalidad de estudios a distancia apoyada en el uso de las TIC, resulta vital para el logro de la autonomía y para el alcance de aprendizajes significativos, determinar los conocimientos actuales sobre las competencias docente en el desarrollo de acciones formativas en un escenario tecnológico, específicamente en el diseño de materiales educativos multimedia.

En tal sentido surge como como inquietud: ¿Cuáles son las competencias cognitivas en el manejo y uso de las tecnologías de la información y comunicación de los profesores del programa de especialización en telemática e informática en educación a distancia de la Universidad Nacional Abierta?

Las ofertas educativas en estudios a distancia a través del uso de las tecnologías de la información y comunicación se han convertido en una poderosa alternativa en el proceso de formación continua de las personas, puesto que al estar sustentado en las TIC los aspectos relacionados con tiempo y espacio físico (coincidencia en un aula de clases) no representan un obstáculo.

Sin embargo, la calidad educativa en el aprendizaje de los estudiantes debe estar garantizada por los materiales educativos utilizados, por cuanto a través de ellos se le brinda al docente una herramienta clave en sus procesos interactivos, indispensables en el aula virtual.

En tal sentido, se requiere de competencias particulares en los docentes que se desempeñan en contextos educativos virtuales, que se deben desarrollar a lo largo de la vida activa del docente, por lo cual Capacho (2011) expone que "...la educación y la formación de personas en cualquier área del saber es uno de los retos más importantes de la Sociedad del Conocimiento para la continuidad de la vida" (p. 27). 
Por su parte, Cabero (2005), citado por Cedeño (2007) ,refiere que la introducción e implementación de las TIC por parte de los docentes, demanda además de actitud positiva por parte de éste, un proceso que permita el desarrollo de competencias que a su vez impulse una adecuada utilización e incorporación por parte del profesor en su práctica educativa.

De igual manera, Zabalza (2007) considera que las competencias involucran las actuaciones prácticas de los docentes en cuanto al saber sobre, saber cómo y el saber operar de manera práctica; así como el ejercicio eficaz de la función docente sumado a sus actitudes en cuanto a formas de actuación, intereses y motivaciones.

En este mismo orden de ideas, visto el rol del docente y su impacto en los niveles de calidad educativa, se hace necesario ante este reto, que dicho docente cuente con un proceso continuo de formación que aseguren las competencias clave, los conocimientos y los aprendizajes que garanticen a su vez, la formación de nuevas generaciones con un desempeño exitoso y efectivo de cara a la sociedad del conocimiento.

Dicha formación implicaría en sus resultados la apropiación de la tecnología, dado que se produce un cambio en la práctica docente al iniciar ellos el uso de las TIC (Celaya, Lozano, y Ramírez, 2010), lo que permite visualizar en esos cambios la incorporación de conocimientos, habilidades y destrezas.

En tan sentido, en una investigación realizada sobre el Programa de Formación Docente del año 2017 de la Universidad Regional Sureste del Estado de México y adscrito a la Dirección General de Asuntos Académicos, se fijó como objetivo establecer las líneas generales para el desarrollo de la formación y actualización de los profesores y las etapas en que ésta se ejecutaría.

Se atendió de manera gradual las necesidades de las Facultades y Escuelas, así como las de los docentes y estudiantes, con el fin de permitir que la labor del docente guíe la formación del estudiante hacia el cumplimiento de la Misión institucional.

Dicha investigación se inició con base en un diagnóstico de necesidades de capacitación que brindó la oportunidad de atender aspectos generales y específicos relacionados con los procesos de enseñanza y aprendizaje, la incorporación de tecnologías y la integración del modelo educativo, todo como base de futuras acciones.

La fase diagnóstica, permitió evidenciar que la formación debe operar como un sistema integral, cíclico y permanente, capaz de generar, en las entidades académicas que conforman la Universidad Regional del Sureste, mejores resultados en el cumplimiento de las funciones que tienen a su cargo.

Además de propiciar en los profesores, el desarrollo de mejores actitudes, habilidades y destrezas, competencias clave, constituyéndose así en un factor relevante de superación laboral, profesional y personal.

Lo interesante es verificar si está vigente el proceso de formación continua del docente para el desarrollo de la capacidad de innovar, que de igual forma se traduce en una competencia para lo cual se requiere estudiar, aprender y reaprender constantemente, de esta manera se pueden incrementar los niveles de investigación y así lograr, entre otras cosas, el diseño y uso de materiales educativos apropiados para el aprendizaje en ambientes virtuales. Así como también sustenta su rol como agente de cambio que hasta puede llegar a impulsar reformas educativas interesantes.

Siendo lo ideal que durante su proceso de formación como docente se incorpore la formación respectiva en cuanto a las TIC, su uso y cómo integrarlas a su ejercicio docente, cómo lograr que formen parte de sus estrategias de enseñanza. Dicha formación en temas de teoría y práctica no debería 
quedarse en sus años básicos de formación, debería continuar a lo largo de su carrera profesional, como proceso continuo de actualización, dada la misma versatilidad y renovación que en sí mismas tienen las TIC, como recurso ineludible en el proceso de enseñanza- aprendizaje de hoy.

Según Fernández, Domínguez y De Armas (2013), los materiales educativos son definidos como "...un recurso en formato digital utilizado en los procesos de enseñanza y aprendizaje, pero también cualquier material digital que un profesor $o$ alumno utiliza para cursar una asignatura: programa, calendario, guía docente, apunte, actividad, tutorial" (p. 27).

Dicho recurso representa un material clave dentro del proceso formativo que se lleva a cabo a distancia por cuanto cataliza el logro de los objetivos, estimulan el desarrollo de habilidades y conocimiento y son considerados de calidad si cumplen con efectividad los criterios didácticos en cuanto a su aporte en los resultados académicos, y tecnológicos con base a su robustez, interoperabilidad, usabilidad y escalabilidad del material.

Los materiales educativos o didácticos contienen la información en diferentes formatos, así como las actividades necesarias que requiere el estudiante, dado la no continuidad y proximidad del docente.

Según Sangra (2006), los materiales educativos permiten al docente:

enfatizar, reforzar y propiciar actitudes, y dinamizar y conducir el proceso formativo". Además, expresa este autor que estos materiales no solo representan un soporte tecnológico, sino que se refieren a actividades formativas o a los contenidos, entre otros, por lo que se considera que intervienen en el proceso de enseñanza $y$ aprendizaje. (p. 239)
En tal sentido, se puede decir que los materiales pueden cumplir varias funciones: servir de guía en cuanto a las actividades que tiene que realizar el estudiante para lograr los objetivos propuestos, promover el aprendizaje en niveles que puedan permitir la metacognición en el estudiante y a su vez la trasferencia logrando significado al aplicarlo en situaciones reales, y fomentar e ilustrar la discusión de lo que ha aprendido.

Por último, el material educativo debe permitir que el estudiante se autoevalúe para determinar la brecha entre lo aprendido y lo que falta por aprender, y generar acciones de mejora, en caso de ser necesario. Por otro lado, fomentar la interactividad, dado el formato no lineal de la información, con lo cual se responde a estilos individuales, así como a sus necesidades de información y conocimiento.

Según Cabero y Gisbert (2002), citado por Muñoz y González (2009), los materiales didácticos deben permitir:

- Aprender a aprender.

- Construir aprendizajes.

- Establecer relaciones entre los diferentes conocimientos presentados.

- Profundizar en los conocimientos.

- Analizar los conocimientos desde diferentes perspectivas.

- Facilitar el control del proceso de aprendizaje.

- Aprender a analizar y aplicar los conocimientos existentes.

- Estimularlo y motivarlo por su presentación y estructura, y

- Facilitar la transferencia de lo adquirido. (p. 36)

Por su parte, Padrón (2009) plantea las características que deben tener los materiales didácticos: carácter reusable en cuanto a capacidad de uso, tanto en diferentes situaciones educativas como en espacios; la calidad en cuanto a valor pedagógico, la usabilidad de su interfaz, así como el 
cumplimiento de estándares internacionales.

En este particular, Sangrá (2006) expresa que desde su estructura los materiales deben tener presente: El módulo como nivel superior, que a su vez está compuesto por unidades. Tales unidades se corresponden a un objetivo específico y los denominados elementos generales, tales como un glosario, la bibliografía, evaluaciones, entre otras.

La transformación de los materiales educativos se ha dado en la misma medida que ha evolucionado las TIC y lo cual ha permitido la creación de materiales educativos con estructuras más complejas, con recursos más sofisticados, con medios de producción distintos; se ha roto el esquema de linealidad, brindándole al estudiante mayor autonomía en su proceso de aprendizaje.

Sin embargo, siempre es importante un constante monitoreo en cuanto a la valoración de la calidad de estos recursos, para de esta manera realizar los ajustes necesarios y ofrecer al estudiante acciones formativas con materiales educativos de elevada calidad.

En tal sentido, Domínguez, y otros (2005) presentan una investigación en la cual realizan un estudio comparativo internacional sobre modelos estandarizados de evaluación y marcas de calidad de materiales educativos digitales. Dicho estudio lo realizaron por etapas, en la primera se centraron en la búsqueda de sellos y agencias de certificación, en la segunda fase analizaron los diferentes modelos de calidad de materiales educativos digitales, y en la tercera, se orientaron hacia la presentación de sus conclusiones y la elaboración de un manual así como de las pautas para el diseño de acciones formativas basadas en materiales educativos digitales reutilizables.

Entre las conclusiones de Domínguez, y otros (2005) se destaca que: "los pilares son, fundamentalmente, los materiales junto con la tutoría, y la atención y satisfacción del usuario como fin último" (p. 14).

De igual manera, los modelos o sellos de calidad que seleccionaron fueron: Comité de la Industria de la Aviación (AICC), American Federation of Teachers (AFT), Athabasca University, National Learning Network, Edna On line Education Network, - IMS Global Learning Consortium, INC y Grant McEwan College.

A partir del análisis de los autores antes citados, se generaron criterios e indicadores de calidad como propuesta para certificar una propuesta de evaluación de calidad de materiales educativos digitales, a partir de un material base para que pueda existir la reutilización de los materiales y generar guías que permitan determinar la forma de articular esos materiales con los programas formativos, de manera tal que de lograr la reutilización y adaptación a diversos grupos de alumnado a fin de responder a sus necesidades y especificaciones.

En este estudio se destaca la importancia de cumplir ciertos sellos o estándares internacionales, información que presentan en dicha propuesta y que permite ejercer el acto evaluativo de los materiales para su adecuación con las actividades, contenidos y objetivos de la asignatura en la que se utiliza. Siendo los materiales educativos un invalorable recurso en el aula virtual debido a que facilitan en el estudiante el logro de su aprendizaje, dichos materiales de enseñanza deben ser muy bien planificadas por el docente previo a su ejecución.

La necesidad de los materiales educativos viene dada por su carácter instrumental para realizar la acción formativa, su función es canalizar el proceso de aprendizaje-enseñanza a través de: apoyar en el logro de los objetivos, facilitar al estudiante la adquisición de nuevos conocimientos, generar nuevas habilidades y 
nuevas actitudes sobre una materia; posibilitar al estudiante el aprender a aprender. Además, como aspecto resaltante, dichos materiales permiten construir el aprendizaje en los estudiantes sin reducir la actividad mental del mismo a una simple memorización, a lo repetitivo o a lo mecánico.

En estos materiales didácticos el docente busca añadir situaciones que le permitan al estudiante desarrollar las competencias requeridas según el objetivo a lograr, acercarlos a su realidad, los motiva y le agrega, además, un elemento fundamental a la educación a distancia como lo es la interacción. La interacción a su vez debe implicar cierto grado de libertad para otorgarle apertura al estudiante para que decida el camino que quiere seguir; que brinde acceso a materiales complementarios y que a su vez genere intercambio de experiencias y aprendizajes con sus compañeros y su tutor.

Tal interacción se puede dar en función del tipo del material educativo y, en cuanto a clasificación, existen muchas, bien sea por la tecnología utilizada, por la presentación física, el tipo de objetivos, el nivel de interacción, entre otras. Según López (1981), citado por Aguilar, Ayala, Espinoza y Zarco (2014) los materiales educativos se clasifican en:

- Impresos: libros de texto, cuadernos de ejercicios, manuales.

- Equipos: grabadora, televisión, radio.

- Material manipulable: globos terráqueos, microscopios, instrumentos.

- Maquetas y modelos físicos.

- Audiovisuales e informáticos: software, animaciones, simuladores.

- Calculadoras, presentaciones, enciclopedias digitales.

Otra clasificación que resultó interesante a la luz del presente trabajo es la ofrecida por Marqués (2011), citado por Aguilar, Ayala, Espinoza y Zarco (2014), dado a que involucra directamente el aspecto tecnológico y materiales que pueden ajustarse a un aula virtual, en particular los audiovisuales y nuevas tecnologías.

Cuadro 1. Clasificación de los recursos educativos

\begin{tabular}{ll}
\hline \multicolumn{1}{c}{ Criterios } & \multicolumn{1}{c}{ Recursos } \\
\hline \multirow{3}{*}{$\begin{array}{l}\text { Materiales } \\
\text { convencionales }\end{array}$} & Tmpresos (textos): libros, fotocopias, periódicos, documentos... \\
& Materiales manipulativos: recortables, cartulinas... \\
& Juegos: arquitecturas, juegos de sobremesa... \\
& Materiales de laboratorio... \\
Materiales & Imágenes fijas proyectables (fotos): diapositivas, fotografías... \\
audiovisuales & Materiales sonoros (audio): casetes, discos, programas de radio... \\
& Materiales audiovisuales (video): montajes audiovisuales, películas, videos, \\
& programas de televisión... \\
& Programas informáticos (CD, on-line) educativos: videojuegos, lenguajes de \\
& autor, actividades de aprendizaje, presentaciones multimedia, enciclopedias, \\
animaciones y simulaciones interactivas... & Servicios telemáticos: páginas web, weblogs, tours virtuales, webquest, caza \\
Nuevas tecnologías & del tesoro, correo electrónico, chats, foros, unidades didácticas y cursos on- \\
& line...TV y video interactivos. \\
\hline
\end{tabular}


En relación a los materiales digitales, Area (2009), citado por Aguilar, Ayala, Espinoza y Zarco (2014), expresa que:

las tres principales características de los materiales digitales son: 1 ) la presencia del hipertexto como un sistema hipotético de organización de documentos no secuenciales; 2) el concepto de la multimedia [...] que permiten integrar simultáneamente diversos formatos de información: textual, gráfica, audio y video; 3) la interactividad que se puede definir como el nivel de respuesta de la máquina que permite al alumno un cierto grado de control sobre el proceso de aprendizaje. (p. 77)

La clasificación y características de los materiales digitales permiten al docente de acuerdo a su funcionalidad, versatilidad y estrategia docente, seleccionar entre uno $\mathrm{u}$ otro, en función de los objetivos, contenidos y potenciales usuarios de los mismos. Esto da un valor interesante a los materiales educativos seleccionados para el proceso de enseñanza, por cuanto es a través de estos medios que logra el docente interesarse, motivarse, desarrollar habilidades y destrezas, aprender, transferir y compartir con sus compañeros.

En definitiva, los materiales educativos pasan a ser un aliado del docente en su gestión pedagógica, puesto que fomentan la interactividad al facilitar el acceso no lineal a la información, la interconexión, además de permitir la bidireccionalidad en las comunicaciones.

Dado a que el aprendizaje a distancia apoyado en el uso de las TIC ha tomado un auge significativo, se hace necesario conocer e identificar las características que debe presentar un curso virtual para ser considerado como una herramienta de aprendizaje de calidad, particularmente con una variable influyente en el proceso de enseñanza -aprendizaje en red, como lo es los materiales educativos dentro del aula virtual.

Más aún, si se considera el aprendizaje como un proceso activo por parte del estudiante, quien, a partir de sus concepciones previas, sus experiencias en el aula virtual dada por los diferentes niveles de interacción, concentrados en unos objetivos y toda una malla de relaciones, soluciona situaciones y genera su aprendizaje significativo.

Según lo señalan Garrison, Anderson y Archer (2001), citado por Santoveña, (2012), existe una integración evidente entre el pensamiento crítico, la interacción social y el aprendizaje profundo, cuando el proceso es guiado por un docente en espacios virtuales donde se destaca el rol de los materiales educativos desarrollados con tecnología multimedia, dado que los mismos representan un elemento clave para lograrlo.

Por otro lado, los materiales educativos permiten al estudiante adquirir un aprendizaje significativo, puesto que sus contenidos y estructura son los potenciadores del mismo. Sin embargo, situar la tecnología sobre la pedagogía y la didáctica conduce a graves errores, por lo cual se debe considerar lo que Cabero y Román (2008), citado por Carrillo (2014) denominan variables críticas, tales como: competencias cognitivas en el diseño de materiales educativos multimedia del docente de contextos educativos virtuales. 


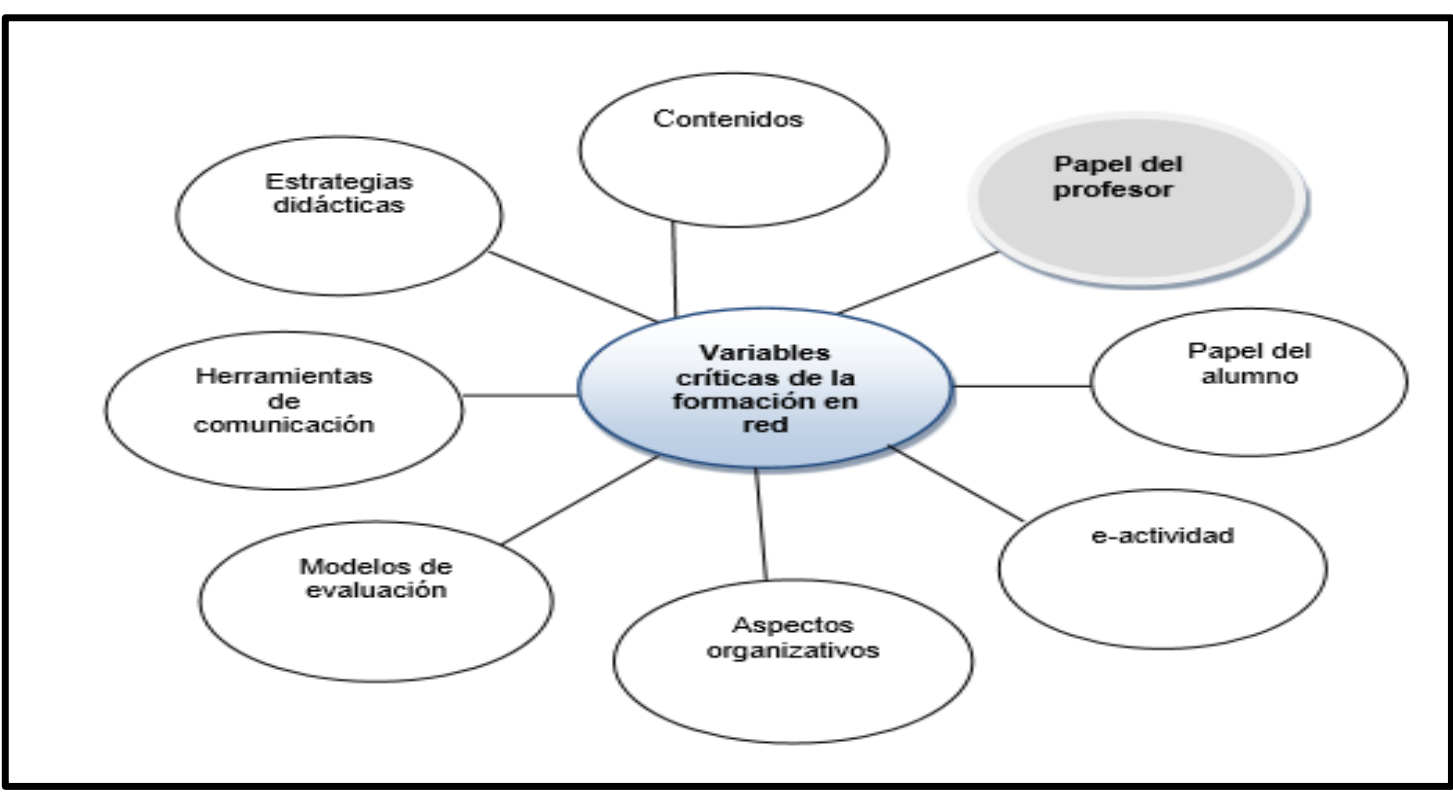

Figura 1. Variables críticas de la formación virtual. (Fuente: Cabero y Román (2008), citado en Carrillo, 2017, p. 38).

Para la presente investigación la variable crítica la representó la referida al papel del profesor en lo atinente a su proceso formativo, el cual permite el desarrollo de competencias para el diseño e implementación de materiales educativos multimedia, por lo que el diagnóstico de tal formación es clave para la subsiguiente toma de acciones en pro de la calidad educativa en los programas de formación de postgrado de la Universidad objeto de estudio.

\section{MÉTODO}

La investigación fue de tipo descriptiva con un diseño de campo, transeccional contemporáneo y una amplitud de foco unieventual. La técnica utilizada para la recolección de datos fue la de la encuesta y como instrumento el cuestionario. Para la validez se utilizó la técnica de juicio de expertos, llevado a cabo por profesores adscritos a otros programas de postgrado de la UNA. La confiabilidad del cuestionario se calculó utilizando el coeficiente de Alpha de Crombach, dando como resultado o,844, por lo que se consideró de alta confiabilidad. La unidad de estudio la conformaron ocho (8) profesores quienes para el momento de esta investigación presentaban como características: estar adscritos a la Dirección de investigaciones y postgrado como subsistema de la UNA encargada de diseñar y desarrollar los programas de postgrado que ofrece la Universidad y tienen más de tres años como experiencia en la facilitación de los diferentes programas de la Especialización. Para el análisis de los datos se utilizó la estadística descriptiva, a través de frecuencias absolutas (fa), frecuencias relativas $(\mathrm{Fr})$, porcentajes y la medida de tendencia central la mediana, la cual se interpretará según baremo expuesto en la Tabla 1. 
Tabla 1. Baremo de interpretación de la mediana

\begin{tabular}{ll}
\hline Categoría & Interpretación \\
\hline o a 3,99 & Muy deficiente \\
4 a 7,99 & Deficiente \\
8 a 11,99 & Regular \\
12 a 15,99 & Suficiente \\
16 a 20 & Excelente \\
\hline
\end{tabular}

\section{RESULTADOS}

Con relación al evento competencias cognitivas en el diseño de materiales educativos multimedia del docente de contextos educativos virtuales, en la Tabla 2 se observa que la mediana se ubicó en 14 puntos, en una escala de 20 puntos, de esta manera se visualiza que éstas competencias, según el baremo de interpretación, se ubican en la categoría suficiente en cuanto a nivel de formación alcanzado por los docentes.

Tabla 2. Mediana del evento competencias cognitivas en el diseño de materiales educativos multimedia del docente de contextos educativos virtuales

\begin{tabular}{lcc}
\hline & & $\begin{array}{c}\text { Puntaje transformado } \\
\text { competencia cognitiva }\end{array}$ \\
\hline $\mathrm{N}$ & Válidos & 8 \\
Mediana & & 14,0 \\
Mínimo & & 9,33 \\
Máximo & & 17,33 \\
Percentiles & 25 & 12,0 \\
& 50 & 14,0 \\
& 75 & 16,0 \\
\hline
\end{tabular}

En la Tabla 3 y gráfico 1 se observa que el $50 \%$ de los docentes tienen, según el baremo de interpretación, las competencias cognitivas en el rango suficiente y un 37,5\% en excelente. Ante lo cual se infiere que estos docentes poseen cierto nivel de conocimiento sobre el sobre el diseño de materiales educativos multimedia; manejan algunos términos $\mathrm{y}$ conocen algunas herramientas, tienen información de ellas y pueden llegar a comprenderlas. En tanto que un 12,5 se ubicó, según el baremo de interpretación, en categoría regular, es decir tiene competencias cognitivas regulares sobre el diseño y producción de materiales educativos multimedia.

Tabla 3. Categorías de las competencias cognitivas

\begin{tabular}{llcccc}
\hline & Frecuencia & Porcentaje & $\begin{array}{c}\text { Porcentaje } \\
\text { válido }\end{array}$ & $\begin{array}{c}\text { Porcentaje } \\
\text { acumulado }\end{array}$ \\
\hline Válidos & Regular & 1 & 12,5 & 12,5 & 12,5 \\
& Suficiente & 4 & 50,0 & 50,0 & 62,5 \\
& Excelente & 3 & 37,5 & 37,5 & 100,0 \\
& Total & 8 & 100,0 & 100,0 & \\
\hline
\end{tabular}




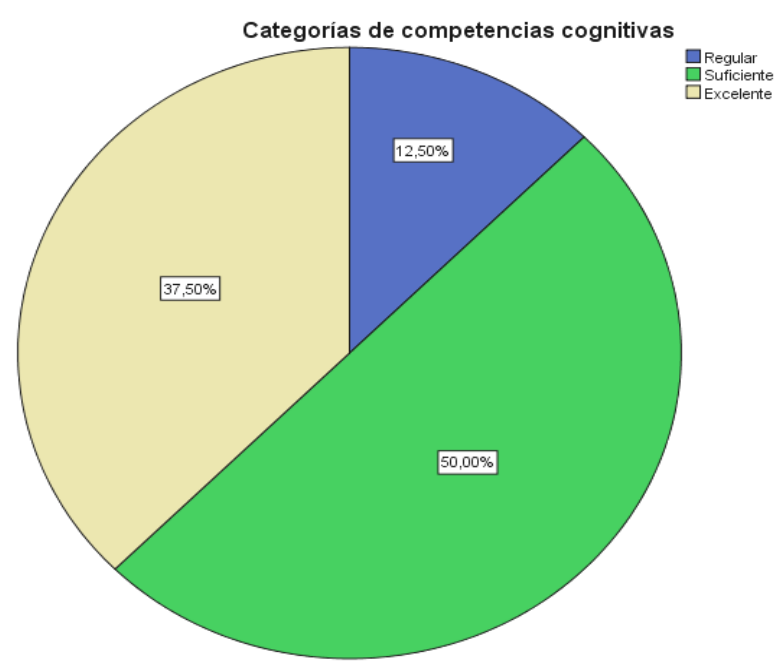

Gráfico 1. Frecuencias y porcentaje del evento competencias cognitivas en el diseño de materiales educativos multimedia del docente de contextos educativos virtuales.

Con relación al evento competencias cognitivas en el diseño de materiales educativos multimedias, a través del análisis de los ítems se observó lo siguiente: el ítem 1 se refiere a los aspectos pedagógicos que se contemplan en las Teorías de aprendizaje, el ítem 4 sobre tipos de contenidos y el ítem 6 cuando considera posibilidades técnicas y pedagógicas. Los profesores de la muestra son docentes de profesión y manejan el contenido al cual se refieren los ítems antes citados. Asimismo, puede apreciarse que el ítem con menor acierto fue el 5 , relacionado específicamente con aspectos netamente técnicos.

De igual manera, se visualiza que el ítem 2, en el cual se obtuvo el $100 \%$ de respuestas acertadas, estuvo referido con el área de conocimientos sobre las Teorías de aprendizaje, lo que tiene relación al obtener respuestas acertadas en un $75 \%$ en el ítem 1, según se observa en el gráfico 2 .

ॠ Incorrecto $\square$ \% Correcto

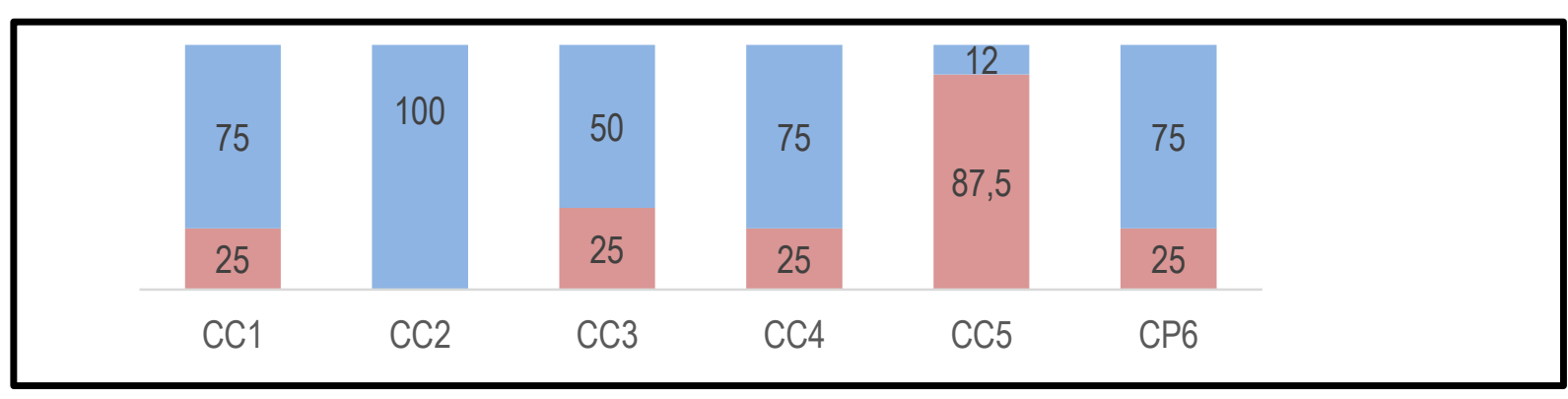

Gráfico 2. Porcentaje de los ítems dicotómicos en el evento competencias cognitivas.

1. Cuando considera los destinatarios del material educativo examina requerimientos pedagógicos

2. La teoría de aprendizaje que enfatiza en el entorno de aprendizaje y en los estudiantes es constructivista
3. La principal utilidad de las herramientas de autor es crear, publicar y gestionar *Mem.

4. Los tipos de contenidos que pueden desarrollarse en un Mem son texto, gráficos y sonido 
5. Cuando contempla tamaño del fichero, capacidad de animación..., atiende características tecnológicas

6. Al momento de seleccionar un material didáctico en la red considera posibilidades técnicas y pedagógicas

* Mem $=$ Materiales educativos multimedias

En el ítem 24, referido a los beneficios de las herramientas de comunicación, el 62,5\% acertó en la respuesta, y un $25 \%$ lo hizo de manera parcial, según se aprecia en el Gráfico 3. Se destaca las herramientas de comunicación como eje central en la modalidad de estudio a distancia apoyada con las TIC.

Se reafirma, en el ítem 21 del tipo abierto lo señalado anteriormente con el ítem 2, del tipo dicotómico, en cuanto a conocimiento de los docentes de las Teorías de aprendizaje, en este caso el ítem 21 es el que logra mayor cantidad de aciertos, en un $75 \%$.

\section{$\square$ Incorrecto Parcial Correcto}

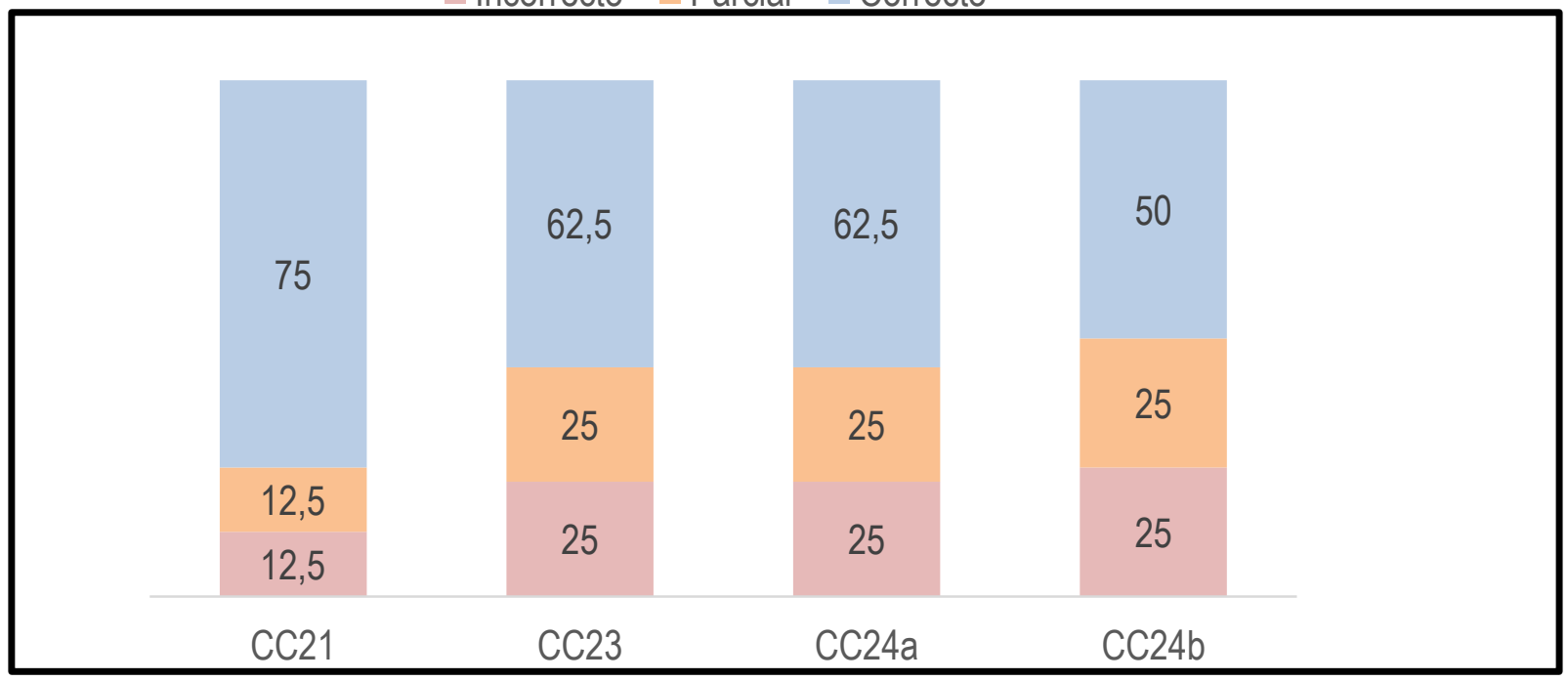

Gráfico 3. Porcentaje de los ítems abiertos en el evento competencias cognitivas.

21. Conoce desventajas y ventajas de usas de teorías de aprendizaje para crear material educativo

23. Sabe los pasos para Insertar un video de YouTube en su aula virtual

24a Conoce beneficios de las herramientas de comunicación que ofrece la Web.

$24 \mathrm{~b}$ Conoce limitaciones de las herramientas de comunicación que ofrece la Web.

Asimismo, se aprecia en el gráfico 3, en un $25 \%$ respuestas incorrectas en la misma proporción en los ítems 23, 24 a y 24 b, dichos ítems si bien se refieren a lo cognitivo se enmarcan dentro del área producción. Por ello no se evidencia en las aulas materiales educativos multimedia de su autoría.

Por otra parte, el conocer en este caso lo vemos en sus respuestas más notorias en lo acertado, que se enfoca hacia los aspectos teóricos de las teorías de aprendizaje, ahora bien, como lo expresan Rubio y Valencia. (2017), "el tipo de problemas que se presentan tiene que ver con el dominio técnico de un software o el manejo de una plataforma...” (p. 111). 
De igual manera, los autores antes citados, resaltan la necesidad de actualización docente sobre todo en temas relacionados con "manejo de ayudas audiovisuales, transmisión de la información y conocimiento mediado por TIC, la forma de hacer una presentación y cómo manejarla con fines pedagógicos" (p. 111).

En razón de lo planteado, la formación del docente para el desarrollo de sus competencias en lo cognitivo, deben apuntar como lo expresó Méndez y otros (2007), a: "los criterios válidos para la selección de materiales, así como conocimientos técnicos suficiente para permitirle rehacer $y$ estructurar de nuevo los materiales existentes en el mercado para adaptarlo a sus necesidades, como crear otras totalmente nuevas" (p. 40).

En efecto, se demanda el replanteamiento de las funciones docentes que se desempeñan bajo la modalidad a distancia apoyada con las TIC en cuanto al establecimiento de competencias que deben alcanzar para su desarrollo integral y uso tecnopedagógico de las herramientas que ofrece la web, que puedan ser compatibles con la tecnología utilizada por la universidad, en busca de la innovación para la adecuación a la sociedad actual.

Lograr el perfil docente demandado requiere una formación continua para el desarrollo de sus competencias orientadas hacia el aprendizaje significativo, en el manejo y uso de las TIC y en el diseño e implementación de materiales educativos multimedia, puesto que, según los resultados obtenidos en esta investigación, tal desarrollo de competencias en ese ámbito no es significativo y al no tener una constante formación no se evidencia cambios, y por ende, no se introducen innovaciones en el currículo.

Se destaca que el proceso formativo de los docentes que se desempeñan en la modalidad a distancia apoyados con las TIC, debe realizarse en términos tanto teóricos como prácticos, en temas tecnológicos como pedagógicos en cuanto uso tecnopedagógico de las TIC en las aulas virtuales, al considerar lo expuesto en el sentido que la herramienta por sí sola no garantiza elevados niveles de motivación ni de aprendizaje, más aún cuando se afirma que se está en la sociedad del aprendizaje, lo que lleva implícito la necesidad de formación continua.

Además, los materiales se convierten en una estrategia de enseñanza para el logro de los aprendizajes en los estudiantes; son considerados como factor de éxito, según lo relatan Cabero y Gisbert (2005). Ahora bien, para cubrir criterios de calidad en el proceso de diseño y producción se requiere de ciertos conocimientos, habilidades y destreza; lo cual, acompañado de políticas institucionales dirigidas a la motivación del docente, hacia el apoyo desde diferentes perspectivas para lograr el desarrollo de tales competencias que permitan contar con materiales educativos multimedia de vanguardia, cónsonos con las características propias de la Universidad.

Dentro de la dentro de la dimensión pedagógica los materiales educativos deben estar diseñados de manera tal que permitan catalizar exitosamente los aprendizajes en sus estudiantes a través de su tutoría encauzada y dirigida por la dimensión informativa donde estos se encuentran.

En efecto, en la medida que se generen y compartan los materiales didácticos entre los diferentes programas de postgrado, en la universidad se gestará una cultura tanto de aprendizaje innovador, sumergido en una arista de su rol: la investigación, en aras de contar con materiales de calidad didáctica de impacto directo en los aprendizajes en los estudiantes.

Asimismo, el docente se adecúa a las necesidades y características del siglo XXI e 
impacta de manera positiva en la calidad pedagógica, al agregar valor otorgado en su capacidad para diseñar materiales educativos multimedia, donde se estructuran contenidos en función de los recursos y tecnologías disponibles, adecuadas para el logro de los objetivos de aprendizaje.

Por otro lado, el docente se constituye para sus estudiantes, que en su mayoría son docentes en ejercicio, como modelador de conductas efectivas, potenciando a un mayor nivel el aprendizaje en sus estudiantes al ofrecerles materiales que les permitan seguir su ritmo, avanzar de manera autónoma y autogestionada. Además, se requiere apoyar al docente en el proceso de agilizar cambios en sus prácticas educativas, así como respaldar iniciativas que permitan ampliar sus competencias cognitivas.

Los docentes deben estar sujetos a constante procesos formativos que promuevan a su vez la actualización de los materiales que se utilizan en los diferentes programas académicos. Con ello se estará a la vanguardia y se influenciará en la calidad educativa de la universidad, y se atiende a la denominada Ley de la vida media de la tecnología de Ed Michaels que establece que "la vida media de la tecnología se hace cada vez más corta" (Cornella, 2004, p. 18).

\section{CONCLUSIONES}

Con respecto a las competencias cognitivas en el diseño de materiales educativos multimedia del docente de contextos educativos virtuales, objeto de este estudio se pudo determinar que según los resultados obtenidos en la investigación el desarrollo de competencias en ese ámbito no es significativo, lo cual se evidencia al no poseer el docente el dominio técnico de softwares o el manejo de plataformas, sus conocimientos, en la mayoría de los casos, se queda en la teoría. En razón de esto, dichos docentes requieren de una formación continua para el desarrollo de sus competencias orientadas hacia el aprendizaje significativo, en el manejo y uso de las TIC y en el diseño e implementación de materiales educativos multimedia.

De igual manera, se puso en evidencia que el proceso formativo de los docentes que se desempeñan en la modalidad a distancia apoyados con las TIC, debe realizarse en términos tanto teóricos como prácticos, en temas tecnológicos como pedagógicos en cuanto a uso tecnopedagógico de las TIC en las aulas virtuales.

Sin embargo, a pesar de que el docente no recibe una constante formación en el diseño, manejo y uso de materiales multimedias, también pudo notarse que un alto porcentaje presenta competencias cognitivas en la materia en referencia, lo cual pone de manifiesto el interés y preocupación del docente en su formación.

\section{REFERENCIAS}

Aguilar, I.; Ayala, J.; Espinosa, O. y Zarco, A. (2014). Análisis de criterios de evaluación para la calidad de los materiales didácticos digitales. Revista CTS, № 25 , vol. $9,73-89$

Cabero, J. y Gisbert, M. (2005). La formación en Internet. Guía para el diseño de materiales didácticos. Sevilla: Eduforma. En https://books.google.co.ve/books?id=$\mathrm{sJrbH}$ 58xjoC\&printsec $=$ frontcover $\& \mathrm{dq}=\mathrm{m}$ ateriales+didacticos\&hl $=$ es\&sa $=X \& v e d=0$ CBwQ6AEwAGoVChMIgvWMjNHoxgIV SMo-

chzkuA_Y\#v=onepage\&q=materiales\%2o didacticos\&f=false

Capacho, J. (2011). Evaluación del aprendizaje en espacios virtuales - TIC. Bogotá: Editorial Universidad del norte

Carrillo, D. (2014). Competencias TIC de los Docentes para la enseñanza mediante Entornos Virtuales en Educación Superior. El caso de la Universidad de Los Andes - Venezuela: Evaluación y Diseño de un Plan de Formación. Tesis Doctoral. Universidad Rovira I Virgili. Tarrragona. 
En www.tdx.cat/bitstream/10803/285330 /1/Tesi\%2oDayana\%2oBeatriz.pdf

Celaya, R., Lozano, F. y Ramírez, M. (2010). Apropiación tecnológica en profesores que incorporan recursos educativos abiertos en educación media superior. Revista mexicana de investigación educativa, 15(45), 487-513. Recuperado de http://www.scielo.org.mx/scielo.php?scri $\mathrm{pt}=\mathrm{sci} \_$arttext \&pid=S1405-6666201000020 ooo $7 \& \operatorname{lng}=\mathrm{es} \& \mathrm{t} \operatorname{lng}=\mathrm{es}$

Cornella, A. (2004). Infoxicación: buscando un orden en la información. Barcelona: Infonomia

Domínguez, G.; Redondo, S.; Giménez, E.; Lozano, L.; Rodríguez, R.; Segura, B. (2005). Estudio comparativo internacional sobre modelos Estandarizados de evaluación y marcas de calidad de materiales educativos digitales. Modelos de desarrollo, explotación y análisis de calidad para la elaboración del multimedia educativo. Madrid: Centro Nacional de Información y Comunicación Educativa. Recuperado de http://redined.mecd.gob.es/xmlui /bitstream/handle/11162/65829/0o8201120 13537.pdf?sequence $=1$

Fernández, A., Domínguez, E., y De Armas, I. (2013). Diez criterios para mejorar la calidad de los materiales didácticos digitales presentada en el VII Jornada Campus Virtual UCM: valorar, validar y difundir. Campus Virtual. Madrid: Universidad Complutense de Madrid

Guadilla C. (2005). Balance de la década de los 90 y reflexiones sobre las nuevas fuerzas de cambio en la educación superior. En Mollis, M. (Compilador). Las universidades en América Latina: ¿Reformadas o Alteradas? La cosmética del Poder Financiero. (pp. 13-35). Argentina: CLASCO

Méndez, A.: Barceló, A.; Rivas, D. y Borrego, M. (2007). Entornos virtuales de enseñanza y aprendizaje. La Habana: Editorial Universitaria

Muñoz, P y González, M. (2009). El diseño de materiales de aprendizaje multimedia y las nuevas competencias del docente en contextos teleformativos. En: https://books.google.co.ve/books?id=oeg suFHM 5 tMC\&printsec $=$ frontcover $\& d q=m$ ateriales+multimedia\&hl=es\&sa $=X \& v e d=$ oCBsQ6AEwAGoVChMI7JO_qdnoxgIVSx $\mathrm{o}-\mathrm{Ch} 2 \mathrm{GrQlg} \# \mathrm{v}=$ onepage $\& \mathrm{q}=$ materiales\%2omultimedia\&f=false

Padrón, C. (2009). Desarrollo de materiales didácticos desde una perspectiva basada en modelos. (Tesis doctoral) En: http://earchivo.uc3m.esPlastino, A. (2008, febrero). La universidad: lugar de investigación científica y transferencia tecnológica. Revista Educación Superior y Sociedad, nueva época. Caracas: Cendes

Rubio, A. y Valencia, G. (2017). El ecosistema educativo universitario impactado por las TIC. Anagramas -Rumbos y sentidos de la comunicación-, 15(30), 101-120. https://dx.doi.org/10.22395/angr.v15n3oa5

Santoveña, S. (2012). Calidad de la metodología didáctica por medio de entornos virtuales de aprendizaje en la formación de agentes educativos. Contextos educativos. Madrid, España: UNED

Sangrá, A. (2006). Modelo de análisis para la implantación de EAD. En Barberá, E. (Coord.); Romiszowski, A.; Sangrá, A. y Simonson, M. Educación abierta y a distancia. Barcelona, España: Editorial UOC

UNESCO (200o). Marco de Acción de Dakar sobre Educación para todos: Cumplimiento de nuestros compromisos colectivos, adoptado por el Foro Mundial de Educación. Dakar. París, Francia: UNESCO

UNESCO (1998) Conferencia Mundial sobre Educación Superior. La Ecuación Superior en el siglo XXI: visión y acción. París, Francia: UNESCO

Zabalza, M. A. (2007). Competencias docentes del profesorado Universitario: Calidad y Desarrollo Profesional. Madrid: Narcea 